\title{
Mapeamento Sistemático: Metodologias e Ferramentas para a Formação de Professores em Pensamento Computacional
}

\author{
Adrianne Veras de Almeida - UFPA/ICEN - verasadrianne3@gmail.com \\ https://orcid.org/0000-0003-3250-3916 \\ Adrielle Veras de Almeida - IFES/CEFOR - adrielle.veras@gmail.com \\ https://orcid.org/0000-0001-8714-5453 \\ Fabíola Pantoja Oliveira Araújo - UFPA/ICEN - fpoliveira@ufpa.br \\ https://orcid.org/0000-0002-8485-5799
}

\begin{abstract}
Resumo: O Pensamento Computacional é uma estratégia para resolução de problemas através da utilização de habilidades cognitivas na área da Ciência da Computação. Neste contexto, o artigo apresenta um mapeamento sistemático visando a identificação de contextos metodológicos e ferramentas usadas para aprendizagem do Pensamento Computacional para a formação de professores no mundo entre os anos de 2010 a 2021, publicados em periódicos e anais de congressos da área da computação. Como resultado, verificou-se que a ferramenta Scratch e a computação desplugada são as estratégias mais utilizadas por meio de oficinas e cursos. Além disso, buscou-se elencar as instituições e paises que mais publicaram sobre o tema, foram identificadas as principais dificuldades e as conclusões reportadas pelos autores dos 22 estudos mapeados.
\end{abstract}

Palavras-chave: Pensamento computacional, formação de professor, ciência da computação.

\section{Systematic Mapping: Methodologies and Tools for Training Teachers in Computational Thinking}

Abstract: Computational Thinking is a strategy for problem solving through the use of cognitive skills in the area of Computer Science. In this context, the article presents a systematic mapping aiming to identify methodological contexts and tools used for learning Computational Thinking for teacher training around the world between the years 2010 and 2021, published in journals and conference proceedings in the area of computing. As a result, it was found that Scratch and unplugged computing are the most used strategies through workshops and courses. In addition, the institutions and countries that most published on the subject were listed, and the main difficulties and conclusions reported by the authors of the 22 mapped studies were identified

Keywords: Computational thinking, teacher education, computer science.

\section{Introdução}

A computação deixou de ser um campo de conhecimento restrito aos profissionais e cientistas da área, tornando-se parte do cotidiano de todos. Uma das estratégias para a disseminação da computação foi incluí-la como parte do currículo da Educação Básica. O Brasil, apesar de já discutir a inclusão de conteúdos relacionados à computação nas séries iniciais, encontra-se em um cenário distante de conseguir implementar nos currículos escolares, fazendo com que tais conteúdos sejam limitados ao Ensino Técnico ou Superior na área de Computação. 
Diante desta perspectiva computacional, no ano de 2006, a pesquisadora Wing declarou a importância do conhecimento da computação para todos (Wing, 2006). Ela definiu Pensamento Computacional (PC), do inglês Computational Thinking, como um conjunto de competências e habilidades relacionadas aos conceitos fundamentais da Ciência da Computação, cujo desenvolvimento é fundamental, não apenas para cientistas da computação. As pesquisas em seu entorno vêm crescendo tanto no Brasil como no exterior como relatado pela revisão sistemática de (Bordini et al., 2017).

A implementação do ensino de Computação na escola exige professores motivados, dedicados e com competências computacionais, pedagógicas e tecnológicas, a fim de ensinar computação de uma forma que realmente envolva os alunos ( Gal \& Stephenson, 2010; Goode et, at., 2008; Bower et. at., 2017). Diante dessa realidade, é necessário pensar a formação do professor para atuar com o PC. De acordo com Araújo et at. (2015), os conceitos de PC são pouco conhecidos para a maiorias dos professores da educação básica do Brasil. Aliar a aprendizagem de conceitos computacionais no ensino básico pode ser encarado como um desafio no cenário brasileiro, mas possibilita espaço para discussões por parte de pesquisadores e profissionais do ambiente escolar (França \& Tedesco, 2015).

Pode-se destacar também, que a Base Nacional Comum Curricular (BNCC), aprovada e homologada em dezembro do ano de 2018 prevê o desenvolvimento de um conjunto de competências e habilidades ao longo da Educação Básica brasileira com o propósito de preparar os alunos na compreensão e resolução de problemas em situações do dia a dia.

A partir deste cenário, o presente artigo apresenta um Mapeamento Sistemático da Literatura (MSL) internacional, baseado em 18 repositórios nos principais jornais de Informática na Educação, com o propósito de buscar melhor entendimento do cenário atual sobre as pesquisas relacionadas à formação dos professores sobre o $\mathrm{PC}$, visando identificar as metodologias, ferramentas mais usadas e atividades de apoio para o ensino do PC em cursos e/ou oficinas de introdução ao PC voltados aos professores. Em linhas gerais, mapeamentos sistemáticos são estudos detalhados, cujo principal objetivo é a busca por respostas em uma determinada área, procurando diminuir ao máximo o viés do pesquisador de modo a garantir uma resposta fiel e condizente com a realidade (Kitchenham, 2017; Petersen, 2015).

Este trabalho está organizado da seguinte maneira: a seção 2 apresenta os trabalhos correlatos, a seção 3 dispõe dos procedimentos metodológicos do mapeamento sistemático; a seção 4 explana informações gerais sobre os estudos primários, os resultados e discussões. Por fim, são apresentadas as considerações finais e perspectivas futuras desta pesquisa na seção 5 .

\section{Trabalhos correlatos}

A literatura apresenta estudos que buscam identificar iniciativas na disseminação do ensino da computação atrelada ao PC para formação dos professores no mundo. Alinhados a esta visão, o estudo realizado por Ramos et al., (2020) apresenta uma revisão sistemática da literatura com o objetivo de resumir estudos sobre PC para formação inicial de professores. A busca foi realizada em cinco bases de dados de publicações científicas, retornando 100 estudos publicados nos últimos cinco anos e, após aplicação dos critérios, ficaram 50 estudos. Como resultado, obteve-se um conjunto de estratégias ou ferramentas especificamente destinadas a apoiar o aprendizado do PC, tais como: Scratch, Alice e entre outros. Observou-se que o PC se consolida cada vez mais como uma estratégia de 
ensino válida e relevante, não apenas para o ensino/aprendizagem de professores, mas também para outras áreas do conhecimento, incluindo Ciência da Computação.

Por fim, o trabalho dos autores Ausiku \& Matthee (2021) realizou uma revisão sistemática com objetivo de examinar estudos existentes sobre as abordagens de ensino e ferramentas usadas para preparar professores do ensino fundamental para integrar o PC em sua disciplina. Trinta artigos de periódicos e anais de congressos atenderam aos critérios de seleção. As abordagens e ferramentas de ensino que têm sido usadas para treinar professores no ensino de PC nas escolas primárias incluem computação desconectada, robótica, programação.

As percepções dos professores em relação às abordagens de ensino para a integração da PC foram positivas e progressivas após as intervenções. Para apoiar os professores, a maioria dos estudos introduziu módulos no currículo de formação de professores ou em um curso de desenvolvimento profissional para professores em serviço. Os resultados indicaram que a maioria das integrações são feitas em aulas de matemática e ciências. Além disso, a codificação continua sendo uma maneira útil de ensinar PC.

O MSL descrito neste artigo se difere dos acima mencionados pela pesquisa de artigos em periódicos de Computação, em uma janela maior de tempo, buscando relatos de metodologias, atividades, principais conclusões reportadas pelos autores dos trabalhos e as tecnologias utilizadas para prover o ensino e que podem ser replicadas ou adaptadas para a formação continuada de professores em PC.

\section{Metodologia do Mapeamento Sistemático da Literatura}

A presente pesquisa se baseia na execução de um MSL, conforme Kitchenham (2001), "O qual define que o mapeamento é um meio de identificar, mensurar e interpretar toda pesquisa relevante disponível para determinada questão de pesquisa, ou tópico, ou fenômeno de interesse". Na seção seguinte é descrita a construção do protocolo de pesquisa.

\subsection{Definição do protocolo de pesquisa}

Protocolo de Pesquisa é o que descreve todos os passos e métodos necessários para a elaboração do MSL. Para a realização deste estudo foram definidas as etapas apresentadas por Petersen et al., (2018) que são: definições das questões de pesquisa principal e específicas, bases de pesquisa, strings de busca, critérios de inclusão e exclusão, busca dos estudos primários, seleção dos estudos, extração e mapeamento dos dados.

\subsubsection{Questões de pesquisa e específicas}

O intuito de um estudo secundário é identificar estudos primários, extrair e analisar dados destes estudos (Cabrejos et al., 2018). Para isso, é muito importante a construção de uma Questão de Pesquisa (QP) objetiva e precisa o suficiente para ajudar no processo de pesquisa e extração de dados e identificar o estudo da arte das pesquisas que aplicam sobre a formação continuada dos professores atrelado ao PC. Sendo assim, a questão de pesquisa principal deste trabalho foi: (QP): Quais as metodologias utilizadas para o ensino-aprendizagem do pensamento computacional na formação docente? Além da Questão de Pesquisa principal, elaborou-se 5 (cinco) questões específicas (QE) que estão dispostas no Quadro 1. 
Quadro 1. Questões Específicas.

(QE1) Como os estudos distribuem-se ao longo dos anos?

(QE2) Como os estudos estão distribuídos no Mundo?

(QE3) Quais são as atividades e/ou as tecnologias/ferramentas usadas para prover o ensino do PC?

(QE4) Quais dificuldades têm sido apontadas pelos pesquisadores? E como elas foram identificadas?

(QE5) Quais as principais conclusões reportadas relativas à aplicação do PC para formação dos professores?

\subsubsection{Bases de pesquisa e strings de busca}

Considerando a relevância da quantidade e qualidade dos trabalhos publicados na área e eleitas por sua relevância e prestígio na comunidade científica brasileira sobre o PC para formação continuada dos professores, as strings de busca foram aplicadas nos repositórios de estudos, especificados na Tabela 1. Optou-se por utilizar duas strings de busca que foram utilizadas na pesquisa automática, apresentadas no Quadro 2. As palavras chaves usadas foram: computational thinking,Education, tools, teacher training and teacher.

\section{Quadro 2. Strings de busca utilizadas no Mapeamento Sistemático.}

("Computational Thinking" OR "education in Computing" OR "Computing") AND ("tools" OR

"methodologies" OR "techniques" OR "technologies") AND ("teacher training" OR "Teacher training" OR "teacher training" AND "teacher training" OR "teacher training" OR "teacher training") ("Computational Thinking") AND ("Teacher") AND (" Capacity " OR "training")

\subsubsection{Estratégias de busca, critérios de inclusão e exclusão}

Os métodos de busca nas fontes definidas foram: busca automáticas e manuais. A busca automática utilizou-se as strings e na busca manual ocorreu em repositório que não foi possível a aplicação das strings. Após esse processo, os critérios de inclusão e exclusão foram elaborados para fazer a filtragem nos estudos retornados. Dessa maneira, as autoras da pesquisa aplicaram os critérios de inclusão e exclusão definidos individualmente.

Foram estabelecidos dois critérios de inclusão e sete de exclusão. Os critérios de inclusão (CI) foram: (CI-1) Apresenta abordagens para ensino-aprendizagem do PC na formação dos professores; (CI-2) O estudo primário aborda aplicações práticas de ferramentas/tecnologias educacionais para o ensino do Pensamento Computacional para a formação dos professores.

Durante o processo para a seleção dos trabalhos também foram aplicados os seguintes critérios de exclusão (CE), são eles: (CE-1) $\mathrm{O}$ estudo primário não aborda aplicações do ensino-aprendizagem do PC na formação dos professores; (CE-2) O estudo não foi publicado no período de 2010 a 2021; (CE-3) O texto completo do estudo primário está inacessível; (CE-4) $\mathrm{O}$ estudo não está na língua portuguesa ou inglesa; (CE-5) $\mathrm{O}$ estudo apresenta os resultados duplicados, por exemplo, publicado em mais de um evento; (CE-6) O estudo está na modalidade de resumo de keynotes, tutoriais, white papers e artigos incompletos, livros de capítulo, dissertações; (CE-7) É um estudo secundário.

\subsubsection{Seleção dos trabalhos e extração das informações}

Todos os resultados apresentados foram conferidos um por um por duas pesquisadoras para determinar se o artigo deveria ou não ser selecionado no mapeamento. Em caso de discordância sobre a inclusão/exclusão de algum estudo, ficou ao cargo da revisora ( $3^{\text {a }}$ pesquisadora) fazer a filtragem. Um roteiro fora planejado (Figura 1), descrevendo o passo a passo das tarefas que foram executadas, facilitando a organização e melhorando o processo de pesquisa. 


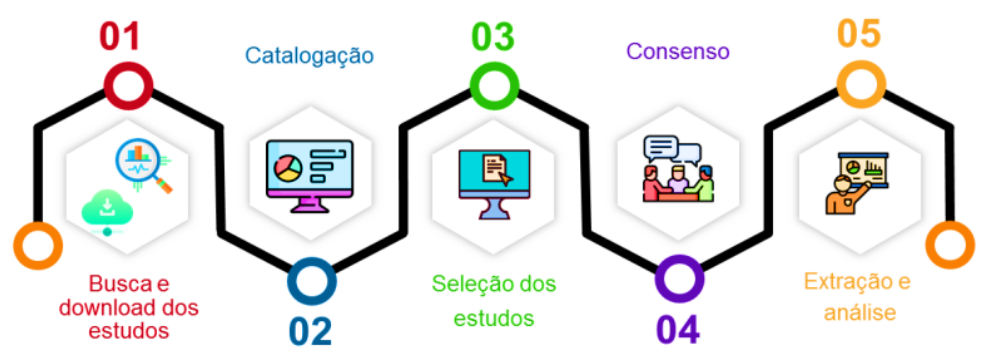

Figura 1. Roteiro do processo de busca.

A Figura 1 apresenta o processo de busca com as seguintes etapas: 1) busca e download dos estudos nas bases de dados; 2) Catalogação dos estudos no programa Parsifal; 3) Seleção dos estudos, cada pesquisadora fez a leitura individualmente do título e abstract; 4) realizou-se o consenso dos estudos que tinham critérios opostos entre as pesquisadoras, dessa forma, fazendo a leitura completa para pode definir se seriam incluídos ou excluídos; 5) Extração e análise de dados dos estudos.

Realizou-se às buscas em todas as fontes selecionadas e os estudos que não atendiam os critérios de inclusão foram descartados. Os artigos foram catalogados na ferramenta Parsifal ${ }^{1}{ }^{1}$, que permite classificação automática de diversas informações referentes a um estudo, facilitando a quantificação dos estudos manipulados. Esta ferramenta ajuda no processo de Mapeamentos ou Revisões Sistemáticos da Literatura e sua escolha foi pelo fato dela ser gratuita, estar disponível online e apresentar facilidade em compartilhar com todos os pesquisadores as informações do MSL.

A aplicação das buscas nas bases de pesquisa neste mapeamento compreendeu o período entre janeiro de 2010 a fevereiro de 2021, apesar da popularização do termo PC ter ocorrido no ano de 2006 no mundo, o surgimento de estudos nas fontes de busca ocorreu a partir de 2012, retornando 77 artigos dos quais 22 foram selecionados (incluídos) neste estudo. $O$ detalhamento desta etapa está disponível na seção 4 e na Tabela 1. Todas as informações dos artigos filtrados e seus metadados estão organizados na ferramenta Parsifal com os 22 estudos selecionados e os 55 excluídos de acordo com os critérios, foram catalogados com a sigla EP (Estudo Primário) com o número do ID, título, autor e ano e seleção dos critérios em uma planilha disponível no Google drive². Assim, o EP1 a EP22 são os selecionados e EP23 a EP77 foram os excluídos.

\section{Resultados e discussões}

Seguindo as etapas do mapeamento sistemático, nessa seção é mostrada a síntese dos dados obtidos, juntamente com a discussão e análise dos dados inerentes a cada questão de pesquisa. A Tabela 1 mostra a distribuição dos estudos primários agrupados por base de dados com os estudos retornados, excluídos e incluídos.

$\mathrm{Na}$ primeira fase, um total de 77 artigos foi retornado pelos engenhos de busca. Ao final do filtro na última fase, 22 artigos abordavam sobre a formação continuada para professores no ensino do PC. Observa-se que a SCOPUS teve o maior número de estudos publicados relacionados com a string de busca.

\footnotetext{
${ }^{1}$ https://parsif.al/

${ }^{2}$ https://docs.google.com/spreadsheets/d/1FmbikU5XAOi056hxZBDQp6DtmMufHZLt/edit?usp=sharing \&ouid=116017226308873319046\&rtpof=true\&sd=true
} 
Tabela 1. Repositórios e distribuição de publicações.

\begin{tabular}{|c|c|c|c|c|}
\hline \multirow{2}{*}{\multicolumn{2}{|c|}{ Base de Dados }} & \multirow{3}{*}{$\begin{array}{c}\begin{array}{c}\text { Estudos } \\
\text { Retornados }\end{array} \\
2\end{array}$} & \multicolumn{2}{|c|}{ Critérios de Exclusão/Inclusão } \\
\hline & & & \multirow{2}{*}{$\begin{array}{c}\text { Excluídos } \\
1\end{array}$} & \multirow{2}{*}{$\begin{array}{c}\text { Incluídos } \\
1\end{array}$} \\
\hline $\begin{array}{l}\text { ACM } \\
\text { TOCE }\end{array}$ & Transactions on Computing Education & & & \\
\hline IEEE ToE & Transactions on Education & 6 & 2 & 4 \\
\hline T\&F CSE & Computer Science Education & 0 & 0 & 0 \\
\hline JCThink & Journal on Computational Thinking & 0 & 0 & 0 \\
\hline $\mathrm{C} \& \mathrm{E}$ & Computers \& Education & 4 & 2 & 2 \\
\hline IHE & The Internet and Higher Education & 1 & 0 & 1 \\
\hline IEEE TLT & $\begin{array}{l}\text { IEEE Transactions on Learning } \\
\text { Technologies }\end{array}$ & 0 & 0 & 0 \\
\hline TandF CSE & Computer Science Education & 1 & 1 & 0 \\
\hline TandF FILE & Interactive Learning Environments & 6 & 5 & 1 \\
\hline BJET & $\begin{array}{c}\text { British Journal of Educational } \\
\text { Technology }\end{array}$ & 0 & 0 & 0 \\
\hline AJET & $\begin{array}{c}\text { Australasian Journal of Educational } \\
\text { Technology }\end{array}$ & 3 & 1 & 2 \\
\hline IJETHE & $\begin{array}{c}\text { International Journal of Educational } \\
\text { Technology in Higher Education }\end{array}$ & 0 & 0 & 0 \\
\hline InfEdu & Informatics in Education & 2 & 0 & 2 \\
\hline EJIS & $\begin{array}{c}\text { European Journal of Information } \\
\text { Systems }\end{array}$ & 0 & 0 & 0 \\
\hline ISJ & Information Systems Journal & 0 & 0 & 0 \\
\hline JMIS & $\begin{array}{c}\text { Journal of Management Information } \\
\text { System }\end{array}$ & 0 & 0 & 0 \\
\hline CAIS & $\begin{array}{l}\text { Communications of the Association } \\
\text { for Inform }\end{array}$ & 0 & 0 & 0 \\
\hline SCOPUS & & 48 & 41 & 7 \\
\hline IEEE & & 4 & 2 & 2 \\
\hline & Total & 77 & 55 & 22 \\
\hline
\end{tabular}

4.1. (QP): Quais as metodologias utilizadas para o ensino-aprendizagem do pensamento computacional na formação continuada do docente?

Com o objetivo de responder à questão principal deste mapeamento, realizou-se uma análise dos estudos. Os resultados obtidos, por meio das informações extraídas, são as seguintes que estão identificadas pelo ID: [EP1] Implementação de um modelo de desenvolvimento profissional (DP) para apresentar o PC para professores; [EP2] Realizaram um workshop para treinar o corpo docente $\mathrm{K}-8$ em uma escola rural e vulnerável para desenvolver habilidades básicas do PC; [EP3] Programa que inclui conteúdo do PC que os futuros professores podem utilizar na vida cotidiana e profissional; [EP4] Realizou um workshop de PC para professores de disciplinas STEM do ensino fundamental e médio.

O estudo[EP5] Projeto para preparar professores do ensino fundamental em serviço e em formação para integrar o PC em suas aulas de ciências; [EP6] Um curso de desenvolvimento profissional Scratch online com base na estrutura do MIT PC e, em seguida, implementaram um treinamento online entre 84 professores de escolas de diferentes disciplinas, incluindo chinês, matemática, inglês e ciência da computação; [EP7] Workshops DP em um formato de conferência virtual de uma semana no ensino de PC; [EP8] Três workshops mostrando como incorporar conceitos de computação em cursos STEM existentes com os professores sobre como assimilar a programação e o PC em suas salas de aula. 
No estudo [EP9] Realizaram um DP contínuo do BootUp para compreender como o conhecimento e as crenças de eficácia dos professores do ensino básico para a codificação e o PC; [EP10] Um curso sobre PC e avaliação do seu impacto na compreensão dos professores de formação inicial, bem como sua atitude em relação à computação; [EP11] Foram apresentadas possíveis atividades de aula para o desenvolvimento de habilidades de PC para professores de diferentes disciplinas; [12] Aulas para educadores do ensino fundamental e médio que tem como objetivo ensinar o PC; [EP13] Curso para ensinar programação, PC e os métodos e ferramentas usados no ensino de programação; [EP14] Um curso a distância especialmente desenhado para professores do ensino fundamental aprenderem os conceitos de PC; [EP15] Intervenções de desenvolvimento para ajudar os professores a utilizar PC e programação;

[EP16] Realização de quatro workshops introdutórios sobre PC; [EP17] Oferta de um curso em conhecimento básico da programação para o desenvolvimento do PC junto com a pedagogia; [EP18] Ofertaram um curso para os professores em formação sobre PC; [EP19] Foi realizado uma experimentação empírica com professores que frequentaram um curso de programação e das habilidades de PC; [EP20] Um modelo de curso incluindo ferramentas de coleta de dados para avaliar conhecimento do conteúdo pedagógico dos professores no ensino de computação; [21] Foi disponibilizado um workshop de desenvolvimento profissional de professores com o objetivo de introduzir conceitos, princípios e práticas do PC; [EP22] Um curso para explorar novas abordagens educacionais, principalmente o PC com o objetivo de melhorar as capacidades dos professores no século 21. Dessa forma, as metodologias obtidas contribuíram para que esses profissionais possam utilizar o conhecimento e o aprendizado adquirido nesta formação para aplicar em sala de aula com os alunos. Essas informações estão detalhadas a seguir, por meio das questões de pesquisas específicas.

\section{2. (QE1) Como os estudos distribuem-se ao longo dos anos?}

Ao analisar a quantidade de estudos publicados por ano, pode-se demonstrar e confirmar o que outros autores destacam no que diz respeito ao crescimento de pesquisas de formação para ensino de computação na Educação Básica atrelado ao PC nos últimos anos no mundo. De acordo com os resultados obtidos, os anos de 2010 a 2011 não tiveram estudos, em 2012 foram dois estudos, nos anos de 2013 e 2017 tiveram um estudo cada e a partir de 2018 houve um crescimento no quantitativo de trabalhos.

No entanto, os anos de 2019 e 2020 foram os que mais tiveram publicações, respectivamente com cinco e seis estudos. Percebe-se que durante os anos apresentados não tiveram uma queda no quantitativo de estudos, com exceção ao ano de 2021 pois foram analisados apenas os três primeiros meses do ano (janeiro a março), ou seja, aumentou a quantidade de publicações ao longo dos anos, o que pode ter sido motivado pelo interesse maior na área.

\section{3. (QE2) Como os estudos estão distribuídos no mundo?}

A distribuição dos estudos pelos países foi baseada na localização das instituições de origem do autor principal. No geral, foram identificados 10 países que têm contribuindo para a formação dos professores em PC, são eles: Chile (1), Lithuania (1), Finlândia (1), Portugal (1), Itália (1), Colômbia (2), Turquia (2), Coreia do Sul (1), China (2) e Estado Unidos (10), sendo o último país foi que teve um quantitativo maior de estudos voltado para o PC da formação continuada dos professores. Referente aos continentes dos 10 países dos estudos primários selecionados, foram identificados o quantitativo de quatro continentes: América do Sul (3), Ásia (3), Europa (6) e América do Norte (10). 


\section{4. (QE3) Quais são as atividades desplugadas e/ou as tecnologias/ferramentas usadas para prover a aprendizagem do Pensamento Computacional?}

Outro ponto de investigação desta pesquisa consiste em descobrir as principais tecnologias/ ferramentas e atividades desplugadas usadas para prover a aprendizagem do PC. A Figura 2 evidencia quais foram as mais utilizadas pelos autores nos estudos mapeados. A soma do quantitativo representado no gráfico é maior que o total de 22 estudos selecionados, isso se deve ao fato de que alguns trabalhos combinaram mais de uma tecnologia na metodologia.

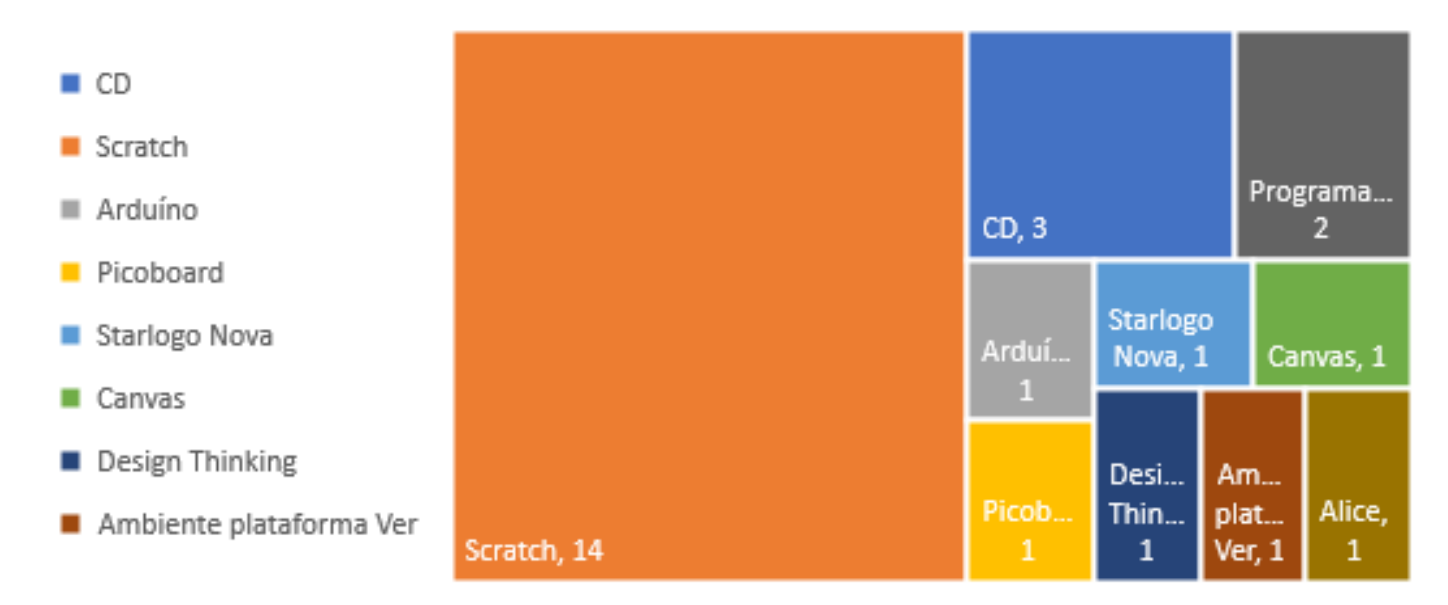

Figura 2. Tecnologias e atividades desplugadas dos estudos mapeados.

Observa-se na Figura 2 que a ferramenta mais utilizada pelos autores foi o Scratch, com o total de 14 vezes, geralmente relacionado à aprendizagem do PC atrelado à linguagem de programação e robótica no desenvolvimento de jogos. A Computação Desplugada (CD) ocupou o segundo lugar e três estudos utilizaram atividades de quebracabeças e torre dos copos, cujo objetivo é construir a torre mais alta e estável fazendo o uso do menor número de copos plásticos em cinco minutos. $\mathrm{E}$ as demais ferramentas/tecnologias utilizadas como: Ver, Arduíno, Picoboard, Starlogo Nova, Alice, Canva, Desing Thinking, foram usados apenas 1 vez.

\section{5. (QE4) Quais dificuldades têm sido apontadas pelos pesquisadores? E como elas foram identificadas?}

Esta questão busca analisar as principais dificuldades existentes nas pesquisas mapeadas. Dos 22 estudos, 20 não mencionaram se tiveram alguma dificuldade na formação. Em apenas dois, os autores relataram os desafios na formação docente na aprendizagem do PC, que estão identificadas pelo IDs [EP1] e [EP17]. No estudo [EP1] um desafio significativo era como desenvolver materiais de aprendizagem que pudessem atender às necessidades de diversos professores (fundamental e médio). No trabalho [EP17], por meio de observação e feedback, alguns participantes demonstraram apenas uma compreensão superficial do pensamento computacional e foram incapazes de criar aulas que integrassem de forma significativa conceitos e ferramentas de PC com conteúdo disciplinar.

\section{6. (QE5) Quais as principais conclusões reportadas relativas à aplicação do Pensamento Computacional para formação dos professores?}

As principais conclusões reportadas nos estudos mapeados refletem um processo de amadurecimento e consolidação do ensino de fundamentos da computação para os 
professores, onde as ações e as práticas tendem a fortalecer os vínculos de interação entre eles e os alunos de forma dinâmica, lúdica e interativa, o que reforça a troca de conhecimentos decorrentes dessas interações construídas sobre diálogos e debates construtivos sob olhares interdisciplinares.

Nesse sentido, apresentou também a relevância do ensino da Computação na escola diante da ausência de referenciais curriculares e profissionais que orientem a prática educacional, sendo a formação em Computação uma alternativa viável para professores de escolas públicas que não possuem os recursos tecnológicos adequados, mas que mesmo assim desejam diversificar suas práticas pedagógicas. Os sujeitos dos estudos reconhecem por meio da formação, programa e capacitação, o alto grau de aplicabilidade dos conceitos computacionais explorados e demonstram compreender a importância do desenvolvimento das habilidades e competências do PC, indicando que esses elementos podem ser facilmente inseridos como meio de condução nas práticas de ensino. Também foi observado nos professores que suas habilidades de pensamento, como resolução de problemas e questionamento foram melhoradas, podendo refletir os conhecimentos e habilidades adquiridos em sua vida diária e profissional.

\section{Considerações finais e direções futuras}

Nesse trabalho realizou-se um MSL com o intuito de conhecer o estado-da-arte sobre o PC para formação dos professores no mundo. Dentre as ferramentas e práticas mais utilizadas na formação, destacou-se o Scratch e CD. Os resultados mostraram um aumento significativo no quantitativo de estudos nos últimos anos no mundo, assim como apontam tendências e a existência da importância do PC atrelados às demais disciplinas do ensino básico à pós-graduação.

Além disso, mostraram que os estudos foram desenvolvidos em 10 países, principalmente nos Estados Unidos, em 10 universidades diferentes, o que evidencia uma grande valorização pela formação dos professores em relação ao PC. Nota-se também que dos estudos mapeados, 10 são do continente da América do Sul. Percebe-se que o número de pesquisas encontradas foi satisfatório, tendo em vista o que descreve este trabalho. Espera-se que este mapeamento tenha fornecido um panorama geral sobre os estudos que utilizaram o PC e seja um esforço inicial para que novas pesquisas e propostas relacionadas a este tema sejam realizadas. Vale ressaltar, que um trabalho realizado nas bases de dados brasileiras abordando este tema foi publicado pelas autoras da presente pesquisa (Almeida et. al, 2021). Para trabalhos futuros, pretende-se complementar este MSL na busca por novos trabalhos, complementando com mais questões norteadoras e realizar busca em outros periódicos na tentativa de ampliar e encontrar mais estudos sobre o tema.

\section{Agradecimentos}

O presente trabalho foi realizado com apoio da Coordenação de Aperfeiçoamento de Pessoal de Nível Superior -Brasil (CAPES) e da Universidade Federal do Pará (UFPA).

\section{Referências}

ALMEIDA, A., ALMEIDA, A., \& ARAÚJO, F. Formação Docente em Pensamento Computacional: Um Mapeamento Sistemático da Literatura. In: Anais do XXIX Workshop sobre Educação em Computação, (pp. 348-357). Porto Alegre: SBC. 2021.

ARAUJO, A. L.; ANDRADE, W.; SEREY, D. Pensamento Computacional sob a visão dos profissionais da computação: uma discussão sobre conceitos e habilidades. In: Anais dos Workshops do Congresso Brasileiro de Informática na Educação. 2015. p. 1454. RENOTE 
AUSIKU, M; MATTheE, M. Preparando Professores de Escola Primária para o Ensino de Pensamento Computacional: Uma Revisão Sistemática. Em: Pang C. et al. (eds) Tecnologias e sistemas de aprendizagem. SETE 2020, ICWL 2020. Lecture Notes in Computer Science, vol 12511. Springer, Cham. 2021.

BOWER, M.; WOOD, L. N.; LAI, J. W.; HOWE, C.; LISTER, R.; MASON, R.; HIGHFIELD, K.; Veal, J. Improving the Computational Thinking Pedagogical Capabilities of School Teachers. Australian Journal of Teacher Education.

BORDINI, A; AVILA, C; MARQUES, M; FOSS, L; CAVALHEIRO, S. Pensamento computacional nos ensinos fundamentais e médios: uma revisão sistemática. In Brazilian Symposium on Computers. In: Simpósio Brasileiro de Informática na Educação. Vol. 28, p. 123.

BRASIL. Decreto n. 8.752, de 9 de maio de 2016. Institui a Política Nacional de Formação de Profissionais da Educação Básica. Disponível em: http://planalto.gov.br/.../Decreto/D8752.htm. Acesso em: 14 de março de 2021.

CABREJOS, L. J. E. R; VIANA, D; Santos, R. P. Planejamento e execução de estudos secundários em informática na educação: Um guia prático baseado em experiências. In: Anais da Jornada de Atualização em Informática na Educação. 2018.

GAL-EZER, J.; STEPHENSON, C. (2010). Computer science teacher preparation is critical. ACM Inroads, 1(1), 61-66. doi: 10.1145/1721933.1721953.

GOODE, J. If you build teachers, will students come? the role of teachers in broadening computer science learning for urban youth. In: Journal of Educational Computing Research. 36(1), 65-88. 2007.

FRANÇA, R.; TEDESCO, P. Um modelo para a aprendizagem do pensamento computacional aliado à autorregulação. In: Anais dos Workshops do Congresso Brasileiro de Informática na Educação. Maceió - AL: CBIE, v. 4, n. 1, p. 85 - 94. 2015.

KITCHENHAM, B; CHARTERS, S. Guidelines for performing systematic literature reviews in software engineering. Technical report, Keele University and Durham University Joint Report. 2007

PETERSEN, K;FELDT, R; Mujtaba, S; Mattson, M. Systematic mapping studies in software engineering. In Proceedings of the 12th international conference on evaluation and assessment in software engineering, volume 17. 2008.

PETERSEN, K; VAKKALANKA; KUZNIARZ, L. Guidelines for conducting systematic mapping studies in software engineering: An update. Information \& Software Technology. 2015

RAMOS, D, X; MAGister, J, A, J; MAGisteR, A, M, D, C; MAGiSTEr, L. Pensamiento Computacional para la formación de maestros: Una revisión sistemática de literatura. 18th LACCEI International Multi-Conference for Engineering, Education, and Technology. .2020.

WING, J. M. Computational thinking. Communications of the ACM, v. 49, n. 3. 2006. 